\title{
Immunofluorescent Characterization of VEGF, VEGFR1 and VEGFR2 Expression in the Corpus Cavernosum of the Diabetic Rat after Prolonged Ingestion of Epigallocatechin Gallate
}

\author{
C. Lombo*, I. Tomada****, C. Morgado***, I. Tavares**** and D. Neves**** \\ * Department of Experimental Biology, Faculty of Medicine of Universidade do Porto, 4200-319 \\ Porto, Portugal \\ ** IBMC- Institute for Molecular and Cellular Biology- Universidade do Porto 4150-180 Porto, \\ Portugal
}

Diabetes type 1 is a very common chronic disease that frequently initiates in youth. Patients suffering from this disease have an increased risk of developing complications such as cardiovascular disease or erectile dysfunction. This is partially due to oxidative stress in affected cells, induced by the increasing production of reactive oxygen species (ROS) that, in addition to oxidative macromolecular damage, inactivate nitric oxide (NO). This condition strongly affects blood vessels of corpus cavernosum (CC), decreasing erectile capability of penis [1]. Thus, we hypothesise that treatment with antioxidants present in the green tea in the initial phase of diabetes type 1 may protect $\mathrm{CC}$ from structural and molecular changes induced by oxidative damage. In support of this hypothesis a previous study of our group demonstrated that green tea long-term ingestion led to decrease in lipid perivascular deposition and vascular endothelial growth factor (VEGF) and its receptor VEGFR 2 expression in CC of aged rats [2]. VEGF is the main angiogenic factor in tissues that induces proliferation and survival of endothelial cells after binding to VEGFR2. In fact, VEGF/VEGFR2 system is involved in both physiological and pathological angiogenic processes, and most of the molecular changes observed in aged $\mathrm{CC}$ derive from oxidative stress coupled to impairment of angiogenic mechanisms. VEGF also engage VEGFR1, nevertheless the exact role of VEGFR1 activation in cavernous tissue is not yet clarified.

Wistar rats were divided into three groups $(\mathrm{n}=8)$ : $\mathrm{C}$ - control group of non-diabetic rats, $\mathrm{STZ} / \mathrm{H}_{2} \mathrm{O}-$ group of rats injected intraperitoneally with streptozotocin solution that receiving water ad libitum, and, STZ/EGCG - group of animals injected with STZ and treated with epigallocatechin gallate, an antioxidant present in green tea $(2 \mathrm{~g} / \mathrm{L})$. The rats were sacrificed 10 weeks after the STZ injection. The $\mathrm{CC}$ was removed and immediately fixed and processed for dual-labelling immunofluorescence detection of the endothelial protein PECAM (platelet/endothelial cell adhesion molecule) and $\alpha$-actin that is expressed exclusively at the smooth muscle cells and, VEGF and their receptors VEGFR1 and VEGFR2. All sections were mounted and observed in an Apotome fluorescence microscope (Carl Zeiss MicroImaging $\mathrm{GmbH}$ ). The morphometrical analysis of smooth muscle content in $\mathrm{CC}$ was also performed, after imunohistochemical detection of $\alpha$-actin (present in smooth muscle), employing the

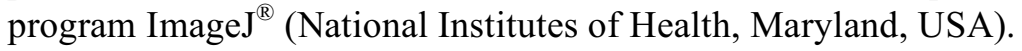

We verified that the expression of PECAM and VEGFR2 was restricted to the endothelium whereas, $\alpha$-actin was expressed at the smooth muscle layer in corpus cavernosum's blood vessels in all experimental groups. On the other hand, the expression of VEGF was observed both in the endothelium and on smooth muscle, often co-localizing with VEGFR1. Morphometrical study demonstrated that $\mathrm{STZ} / \mathrm{H}_{2} \mathrm{O}$ rats presented a reduced layer of perivascular smooth muscle (12.8 \pm 3.5$)$ when compared with that observed in control non-diabetic $(26.2 \pm 3.5, \mathrm{P}<0.05)$ or STZ/EGCG $(23.8 \pm 5.5, \mathrm{P}<0.05)$ animals.

The data here reported suggest that EGCG consumption prevents structural diabetes-induced modifications of the blood vessels in the CC. The role of VEGF needs to be further evaluated namely in the perspective of a target to prevent erectile dysfunction during diabetes.

Acknowledgements: Financial Support of IJUP/UNICER Project 


\section{References}

1. Agarwal et al., J Androl 27: 335-347, 2006

2. Neves et al., AGE 30: 217-228, 2008
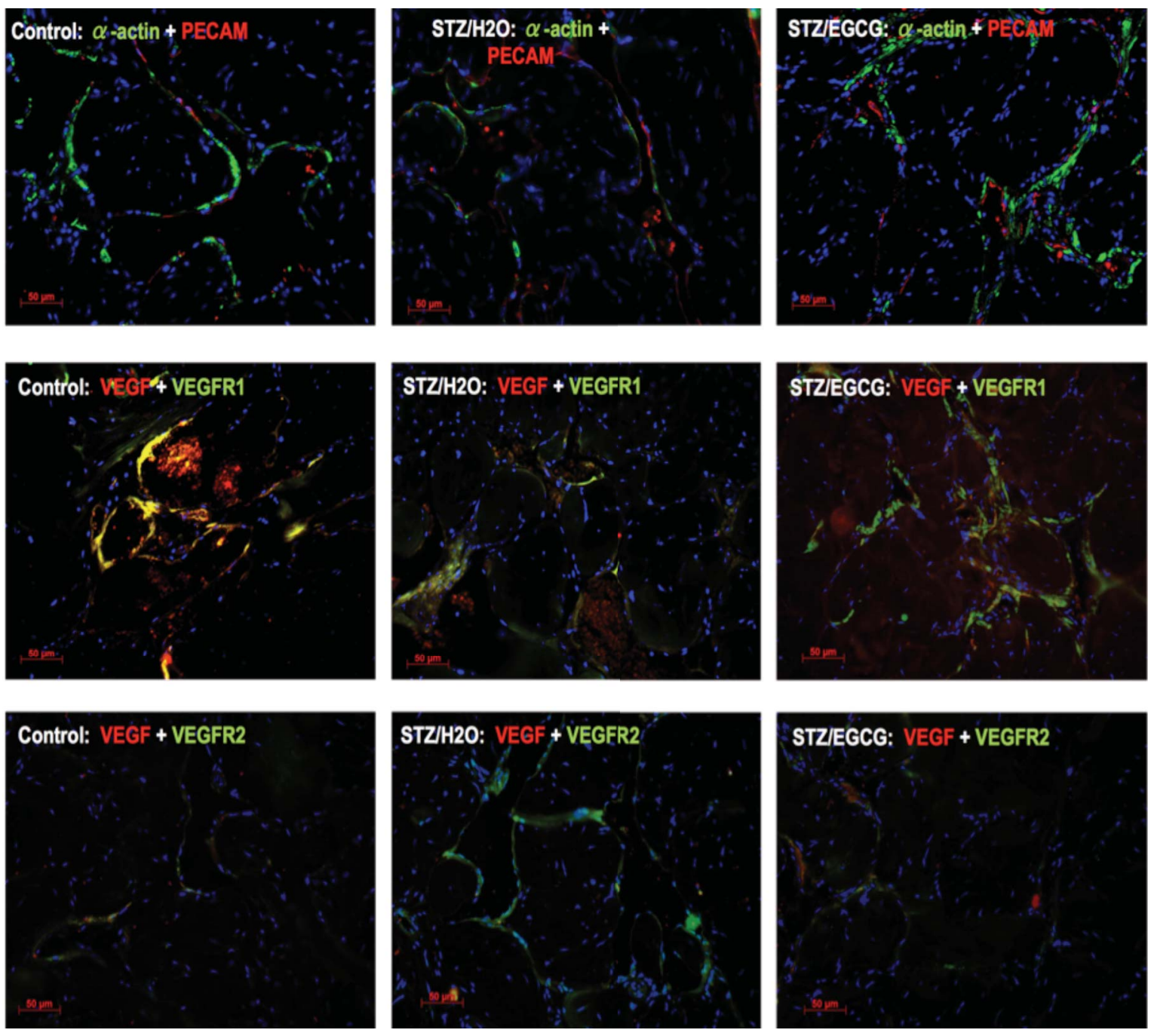

Figure 1. Dual immunolabelling of $\alpha$-actin (green)/ PECAM (red), VEGF (red)/VEGFR1 (green) and VEGF (red)/VEGFR2 (green) in cavernous tissue of rats from all experimental groups. Note colocalization of VEGF and VEGFR1 at smooth muscle cells, and expression of VEGFR2 restricted to the endothelium. Cavernous tissue of rats from group STZ/ $\mathrm{H}_{2} \mathrm{O}$ present a significant reduction in smooth muscle layer thickness, when compared with control and STZ/EGCG groups. 